\title{
LA LABOR EDUCATIVA EN LA UNIVERSIDAD DEL SIGLO XXI: PROYECCIONES PEDAGÓGICAS ACTUALES
}

\author{
Juana María Cubela González, Dra. C. \\ Universidad de Oriente, Cuba \\ jmcubela@uo.edu.cu
}

Palabras claves: labor educativa, estrategias educativas, pautas y
proyecciones pedagógicas.

Keywords: educational work, educational strategies, guidelines and pedagogical projections
Recibido: 18 de Octubre de 2017

Aceptado: 24 de Noviembre de 2017

\section{RESUMEN}

La complejidad del mundo actual y los nuevos retos que tienen que enfrentar los jóvenes universitarios requieren de nuevas miradas de por qué, cómo y hacia dónde dirigir la labor educativa en las universidades. Se exponen nuevos presupuestos teóricos metodológicos que demandan de la utilización de nuevos métodos, estilos, formas y vías para lograr la calidad de esta labor. La Universidad de Oriente (UO) diseña y actualiza de manera sistemática las estrategias educativas desde el nivel de universidad, facultades, carreras, años académicos y grupos, a tono con la realidad social del siglo XXI y el perfeccionamiento que enfrenta la Educación Superior Cubana, apreciándose fortalezas. Se brindan consideraciones, pautas y proyecciones pedagógicas con la intención de continuar logrando trasformaciones en la labor educativa a favor de la formación integral de los estudiantes.

\begin{abstract}
The complexity of the current world and the new challenges that university students have to face require new insights into why, how and where to direct educational work in universities. New theoretical assumptions are exposed - methodologies that demand the use of new methods, styles, forms and ways to achieve the quality of this work. Universidad de Oriente (UO) designs and systematically updates educational strategies from the university level, faculties, careers, academic years and groups, in tune with the social reality of the 21st century and the improvement facing Cuban Higher Education, appreciating strengths. Considerations, guidelines and pedagogical projections are offered with the intention of continuing to achieve transformations in the educational work in favor of the integral formation of the students.
\end{abstract}




\section{INTRODUCCIÓN}

La labor educativa con los jóvenes universitarios requiere de nuevas miradas que se atemperen a la nueva realidad social, política, económica, ética y los desafíos que tienen que enfrentar.

Son muchos los autores que argumentan el porqué, cómo y hacia dónde dirigir los propósitos educativos. Desde estos presupuestos la Educación Superior en Cuba transita por un perfeccionamiento profundo que debe ser asumido con la utilización de nuevos métodos, estilos, formas y vías para lograr la calidad de la formación integral del profesional.

La Universidad de Oriente (UO) consciente de este objetivo proyecta teórica y metodológicamente cómo lograr una labor educativa cada vez más coherente y eficiente.

Es objetivo de este trabajo sistematizar maneras, acciones, pautas pedagógicas y recomendaciones que contribuyan al perfeccionamiento sistemático de las estrategias educativas, a la vez que motiven a los estudiantes a desempeñar un rol protagónico, participativo y potenciador de trasformaciones en su aprendizaje y modos de comportamientos, con una amplia cultura científica, profesional, ética y humanista, comprometidos y preparados para defender las causas justas de la humanidad con argumentos propios y competentes para el desempeño profesional y el ejercicio de una ciudadanía virtuosa.

\section{DESARROLLO}

Los objetivos de trabajo del Ministerio de Educación Superior (MES) para el año 2017 se declaró como estrategia maestra principal el enfoque integral y sostenible para la labor educativa en las universidades.

Consiguientemente, en el Sistema de Evaluación y Acreditación para las universidades cubanas se privilegia, como uno de los aspectos esenciales, la labor educativa vinculada a la calidad de la actuación de los docentes.

Al respecto se precisa que los profesores representan el "conjunto de educadores con elevado compromiso social, lo que se fundamenta en una sólida preparación educativa, científico-tecnológica y profesional, evidenciada en su maestría pedagógica, producción científica y especialmente en su modo de actuación" MES, (2015).

De manera que el perfeccionamiento de la labor educativa es hoy una prioridad para el Ministerio de Educación Superior, lo que debe lograrse desde una visión creadora del "enfoque integral para la labor educativa y político-ideológica en las universidades", el que se caracteriza, en su esencia, por el desarrollo como sistema de todas las influencias educativas que tienen lugar en la comunidad universitaria. 
En la literatura pedagógica, se utiliza indistintamente los términos labor educativa y trabajo educativo, para hacer alusión al mismo fenómeno. Un colectivo de autores del Instituto Central de Ciencias Pedagógicas (ICCP). (2012) puntualiza que la labor educativa es el conjunto de todas las actividades, sin excepción, que en la escuela se realizan dirigidas a alcanzar los objetivos que, de modo integral, confluyen en la formación multilateral y armónica de la personalidad.

Chacón y Báxter (2002) definen el trabajo educativo como labor pedagógica, que tienen la intención de formar, transformar, y educar la personalidad y asumen el trabajo pedagógico como "proceso interactivo e intersubjetivo (relaciones interpersonales directas) que no podrán ser sustituidas por la tecnología".

Por su parte, Makarenko (1971), desde hace más de 50 años, insistió en la necesidad de educar correctamente para no tener que corregir o reformar, tarea que calificaba como difícil y penosa y se refiere al efecto axiomático del ejemplo como el método educativo por excelencia y a la disciplina consciente como resultante de toda labor educativa efectiva, lograda fundamentalmente a partir de la unidad de acción del colectivo.

También subraya que el proceso de educación debe realizarse de manera suave e imperceptible, lo cual no se contrapone con la posición activa del alumno en su educación, además, tiene que tener claridad y perceptibilidad de los objetivos, metas, plazos y las normas de conducta colectiva que deben ser interiorizadas para lograrlo.

La autora Cubela, JM (2016), considera la labor educativa como el quehacer pedagógico del profesor en interrelación e interacción activa con los alumnos para promover de manera intencionada, sistemática, sistémica y coherente el proceso formativo a favor del desarrollo de conocimientos, sentimientos, valores, cualidades, convivencias y modos de comportamientos que sean personalmente significativos en ellos, acorde con los objetivos de su modelo de formación y en correspondencia con sus características socio-física psicológicas - personológicas y la situación socio histórica y contextual que les ha tocado vivir.

En esta definición se hace notorio que solo cuando lo que haga, trasmita y enseñe el docente adquiera sentido personal para sus alumnos y lo marque de manera significativa emocional y afectivamente, podrá convertirse en un dispositivo movilizador de comportamientos y conductas positivas a favor del logro de objetivos personales y la consecución de determinadas metas, aspiraciones y proyectos de vida o profesionales.

En las universidades la labor educativa transcurre mediante diferentes dimensiones o esferas de influencia, las denominadas curricular, extensión universitaria y vida socio-política íntimamente interrelacionadas, aunque particular atención debe tener la curricular, desde el espacio áulico, donde interactúan e interrelacionan cara a cara docentes y estudiantes por un período de tiempo significativo. 
Se debe tomar conciencia de que el actual siglo XXI, caracterizado por la tecnología de vanguardia, la innovación, el auge de la informática, la diversidad, el dinamismo y el acrecentamiento tecno globalizado, impone cambios inaplazables a la labor educativa, si se tiene en cuenta, además, cómo se incrementan las múltiples formas de aprender y de educarse; con iniciativas multi e hipertextuales, virtuales y el intercambio dinámico en redes, que dan cuenta de las diversas maneras de obtener información y conocimientos, y por tanto, implica atender desde nuevas perspectivas transformadoras la labor de educar.

Tampoco puede obviarse la realidad humana, social, económica y valorativa en que se insertan los jóvenes en las sociedades en que viven, aprenden y se desarrollan. La juventud cubana, aunque no está convulsionada directamente por las guerras, las desigualdades sociales extremas, el crimen organizado, el hambre, la exclusión, el narcotráfico y la inseguridad social, entre otras, estas tragedias humanas no dejan de afectarle sensiblemente.

Son otras realidades sociales que cambian, se complejizan y diversifican en la Cuba actual que hay que entender para poder emprender una labor educativa diferente, a tono con lo que piensan, sienten y necesitan los jóvenes, siendo necesarios cambios de enfoques, modelos, estilos y métodos pedagógicos.

A decir de Martínez-Boom (1993), el docente debe redimensionar aquellos saberes imprescindibles para poder realizar una labor educativa coherente y contextualizada teniendo en cuenta:

- El aprovechamiento del contexto áulico y particularmente lo que se enseña en la clase para educar a través de la instrucción.

- El tratamiento, desde los contenidos de las asignaturas, a la cultura escolar (historia, rituales, normas, regulaciones, currículo oculto).

- La profundización en los saberes de los estudiantes (historias personales, familiar, afectiva, escolarizada, grupal de calle).

- La atención a los saberes que tienen que ver con el sentido común (la lógica racional popular).

- El tratamiento a los saberes de la cultura política societal y a los saberes del contexto de cambios (las complejidades y el ritmo acelerado de vida sociocultural de hoy).

Se requiere de profesionales que sepan conjugar su academia con su sensibilidad profesional y sentido de la ética humanizante que sitúa al alumno como el corazón de su quehacer profesional, el cual necesita apoyo, ayuda profesional, solidaridad, respeto y satisfacción de sus necesidades vitales y espirituales crecientes con el desarrollo de buenas prácticas pedagógicas.

Se exhorta la puesta en práctica de la inteligencia emocional del docente, para que pueda influir en el alumno como un ser humano educable en su manera altruista de ver y hacer su propia vida y su contribución a una vida de dignidad humana para todos. Lo que es hoy más apremiante que el bombardeo de información, conocimientos y destrezas que lógicamente deben ser enseñados sobre su profesión. 
El estabelecimiento de una comunicación educativa asertiva y desarrolladora es indispensable en la interrelación de docentes y estudiantes para que logren complementarse, intercambiar saberes, informaciones, sentimientos, valores profesionales y humanitarios y comprenderse mejor mutuamente.

Al respecto, Morin (1999) recordaba que ninguna técnica de comunicación, del teléfono e Internet, aporta por sí misma la comprensión. La comprensión no puede digitarse. Educar para comprender las matemáticas o cualquier disciplina es una cosa, educar para la comprensión humana es otra; ahí se encuentra justamente la misión espiritual de la educación: enseñar la comprensión entre las personas como condición y garantía de la solidaridad intelectual y moral de la humanidad.

A decir del Papa Francisco ante el Congreso de los EE UU el 24 de mayo de 2015, lo cual es genuino para educar, "debemos mirar a las personas, sus rostros, escuchar sus historias mientras luchamos por asegurarles nuestra mejor respuesta a su situación. Una respuesta que sea siempre humana, justa y fraterna.

Cuidémonos de una tentación contemporánea: descartar todo lo que moleste. Tratemos a los demás con la misma pasión y compasión con la que queremos ser tratados. Busquemos para los demás las mismas posibilidades que deseamos para nosotros. Acompañemos el crecimiento de los otros como queremos ser acompañados. En definitiva: queremos seguridad, demos seguridad; queremos vida, demos vida; queremos oportunidades, brindemos oportunidades".

Específicamente, en el tratamiento a los jóvenes alerta que muchos tienen delante un futuro lleno de innumerables posibilidades, muchos otros parecen desorientados y sin sentido, prisioneros en un laberinto de violencia, de abuso y desesperación. Sus problemas son nuestros problemas. No nos es posible eludirlos. Hay que afrontarlos juntos, hablar y buscar soluciones más allá del simple tratamiento nominal de las cuestiones.

Los docentes deben aprovechar los espacios y las oportunidades que brinda el proceso docente educativo (PDE) para: aportar ideas, abrir disertaciones interesantes, dialogar constructiva y trasparentemente para complementar las maneras de pensar, de sentir y de ser de los jóvenes alumnos, ser capaces de hacerlos reflexionar y autor reflexionar sobre las diversas opciones alternativas para construir su crecimiento y proyección personal y profesional a corto, mediano y largo plazo.

Parafraseando a Giroux (1997) los profesores deben reestructurar la naturaleza del discurso, las relaciones sociales e interpersonales del aula y los valores que ellos mismos legitiman en su enseñanza, para poder educar a los estudiantes como ciudadanos activos y críticos, siendo modelos de intelectuales transformativos. 
En esta dirección, valdría la pena atender algunas ideas de los siete saberes que según Edgar Morin deben promoverse desde lo educativo:

- La primera e ineludible tarea de la educación es enseñar un conocimiento capaz de criticar el propio conocimiento.

- El primer objetivo de la educación del futuro será dotar a los alumnos de la capacidad para detectar y subsanar los errores e ilusiones del conocimiento y, al mismo tiempo, enseñarles a convivir con sus ideas, sin ser destruidos por ellas.

- La inteligencia general se construye a partir de los conocimientos existentes y de la crítica de los mismos. Su configuración fundamental es la capacidad de plantear y de resolver problemas.

- La educación debe mostrar el destino individual, social, global de todos los humanos y nuestro arraigamiento como ciudadanos de la tierra. Éste será el núcleo esencial formativo del futuro.

- Enseñar la comprensión significa enseñar a no reducir el ser humano a una o varias de sus cualidades que son múltiples y complejas. Ello implica, no marcar a determinadas personas y grupos sólo con una etiqueta: sucios, ladrones, intolerantes, por el contrario, insistir en las posibilidades de mejorar la comprensión mediante la apertura empática hacia los demás y la tolerancia hacia las ideas y formas diferentes, mientras no atenten a la dignidad humana.

De este modo, se recomiendan algunas maneras de estimular una dinámica de cambios en la labor educativa desde la instrucción, que tenga en cuenta:

$\checkmark \quad$ La flexibilización del proceso de enseñanza aprendizaje, donde a la vez que se enseñen conocimientos se aprovechen las posibilidades de la clase para enseñar aprendizajes de vida.

$\checkmark$ Mayor preparación para enseñar a los estudiantes a aprender a través de su intercambio académico a través de las REDES, aulas virtuales, utilización de repositorios de materiales, bibliotecas virtuales, la utilización del correo electrónico para hacer intercambios académicos con sus docentes u otros docentes y estudiantes.

$\checkmark \quad$ La planificación de tareas integradoras para aprender a partir de tareas productivas en equipos y subgrupos de cooperación y colaboración.

$\checkmark \quad$ La sensibilidad profesional para conocer a través del diagnóstico personalizado del alumno las causas que gravitan en las dificultades en su formación y brindar orientaciones educativas profesionales y oportunas.

$\checkmark$ La toma de la conciencia de la importancia de la profundización en la caracterización personal de los alumnos y a los saberes de los estudiantes: historias personales, familiar, afectiva, escolarizada, grupal de calle.

$\checkmark \quad$ La participación activa, emprendedora y entusiasta de los profesores junto a sus estudiantes en la realización de aquellas actividades trascendentes de tipos curricular, extracurriculares y sociopolíticos con actitudes éticas y fraternas.

En el desempeño profesional como profesores no podemos seguir asumiendo lo educativo como algo abstracto y exterior al proceso de enseñanza aprendizaje. 
Al contrario, en el arte de educar tanto los educadores como los educandos experimentan formas de vida y de relaciones interpersonales marcadas por la historia personal, social y contextual, influenciada por la complejidad y diversidad de sucesos, hechos, acontecimientos que impactan en las maneras de ser y hacer de cada cual, donde no pueden regir los estereotipos, la unilateralidad de pensamientos, la rigidez pedagógica, sino considerar la múltiples posibilidades, opciones y vías alternativas para enseñar valores y comportamientos éticos. Se comparte que no existe una crisis de valores en los jóvenes, sino ganar comprensión de que estos naturalmente cambian de una época a otra, a tono con el momento histórico de que se trate y los intereses generacionales. Se asume la máxima de que la juventud no se ha perdido a sí misma. Lo que generalmente hace es seguir a sus mentores, que ya han avanzado por un rumbo no perfecto y sí muchas veces equivocado.

Por eso, para enfrentar y resolver el tema de los valores, que es como si dijéramos el futuro del país, se hace necesario, entre otras exigencias, comenzar por rectificar el comportamiento de los educadores, el camino en lo económico, con sus correspondientes consecuencias en lo educativo, lo administrativo, lo productivo, lo constitucional y lo judicial, pues habrá que restablecer el orden social general, para poder enrumbar a la sociedad en su conjunto, partiendo de la máxima pedagógica de aprovechar las buenas prácticas, o sea, las experiencias positivas y negativas acumuladas hasta hoy y nuestras propias características y condicionantes.

Otra mirada que hay que enfocar en la labor educativa con la juventud es la educación a favor del amor a los símbolos identitarios y el desarrollo de una cultura autóctona que pueda, desde posiciones valorativas críticas, hacer frente a la invasión de símbolos externos y la guerra cultural, pensada desde modelos extranjerizantes, imperialistas y neoliberales basadas en valores e historias foráneas. Se requiere de una visión antropoética que conduzca a la comprensión de los nuevos significados, signos y sentidos que hay que reconfigurar en la vida actual. Se trata de no perder de vista algunas apreciaciones educativas, a partir de lo que desean y quieren los jóvenes de hoy:

- Hacer creíble el estereotipo del maestro cubano como ejemplo de figura paradigmática del deber ser.

- Promover la innovación de productos colectivos identitarios auténticos donde ellos participen.

- Originar una épica novedosa que involucre a la juventud propiciando su participación activa y protagónica, de manera tal, que promueva el interés por sus símbolos nacionales, por lo que auténticamente distingue a su país como único e irrepetible.

- Establecer una marca de país emanada de cómo lo ven, lo sienten y les interesa, para que puedan portar con orgullo lo que somos popularizando y visibilizando nuestros generosos y altruistas valores.

- Dialogar, intercambiar y crear desde la escucha activa y los lenguajes que se parezcan a ellos.

- Estimular su contribución al desarrollo de una industria cultural suficientemente fuerte que se construya con el talento de los creadores más jóvenes.

- Promover la receptividad crítica de los productos culturales que llegan por diferentes vías, desde la resignificación de nuestros valores como nación, en contraposición a la banalidad, la hegemonía, la invasión, la dominación y la seducción cultural. 
Por lo tanto, sería interesante tener en cuenta algunas pautas pedagógicas para desarrollar la labor educativa en las instituciones educativas:

> Mostrar una profesionalidad en permanente mediación con la firmeza intelectual, el dominio académico y la pertinencia histórica, ética y cívica a favor de una educación más justa, participativa y progresista.

$>$ Incentivar procesos pedagógicos dinámicos, cambiantes y contingentes a favor del alumno.

$>$ Crear espacios académicos para el ejercicio del pensamiento reflexivo, en los que a cada estudiante les sea permitido opinar, pensar, discrepar y aportar acerca de su propia conciencia democrática participativa y lo que puede hacerse institucionalmente para que su formación integral sea mejor.

$>$ Garantizar el derecho al crecimiento individual, a ser incluido, a participar, ser escuchado y ser protagónico.

$>$ Crear, entre todos, un ambiente institucional caracterizado por una demanda ética solidaria, empática, entusiasta y emprendedora que incentive la organización, la estética, la armonía y el bienestar de todos los que conviven en cada espacio escolar.

$>$ Respetar al alumno, tanto en su diferencia como en su identidad personal con comprensión humana.

> Exigir que el quehacer pedagógico formativo se distinga por la información veraz y la comunicación educativa asertiva, como dispositivos claves para el desarrollo de competencias cognitivas, afectivas, sociales y éticas del hacer de la enseñanza.

Atendiendo a los presupuestos teóricos expuestos, en las estrategias educativas que se diseñan ejecutan, controlan y evalúan en las facultades, carreras, años académicos y grupos estudiantiles debe considerarse lo sistematizado con anterioridad, de manera tal, que se conviertan realmente en un instrumento esencial para dirigir el proceso de formación integral del alumno en todos los escenarios universitarios.

Este propósito debe ser atendido pedagógicamente desde todos los escenarios educativos, o sea, todos los espacios en que transcurre el proceso de formación de los estudiantes en la universidad: el aula, un taller, una empresa, un museo, una entidad de cultura, un centro de trabajo donde labore por un período de tiempo para dar cumplimiento a una tarea docente, laboral, de investigación o de impacto y en los escenarios priorizados para influir en los aprendizajes de vida como la residencia estudiantil, la participación en el trabajo comunitario, el área deportiva, el teatro y otros contextos donde realizan las actividades socio políticas.

Consecuentemente, con lo abordado se hacen recomendaciones pedagógicas para el perfeccionamiento sistemático de las estrategias educativas en carreras y años académicos con vistas a la proyección de acciones que motiven:

- La estimulación de modos de comportamientos que se corresponden con un joven honesto, decente y disciplinado en los diversos escenarios y actividades en que participan, manifestados en la puntualidad, disciplina, cortesía, la comunicación asertiva y pertinente y la crítica oportuna y respetuosa.

- La participación activa en las actividades curriculares y extensionistas con la designación de responsabilidades distribuidas, compartidas y designadas a todos 
y cada uno de los estudiantes de la brigada acompañados de sus profesores, con la concreción de fechas determinadas y la asunción de roles diversos que promuevan el liderazgo estudiantil compartido.

- La elevación de la calidad de los resultados de los indicadores de promoción y el aprendizaje autónomo, protagónico, crítico y desarrollador del estudiante.

- La autogestión de su aprendizaje con el uso racional y eficiente de los recursos existentes: plataformas interactivas en las tareas para el trabajo independiente desde la wi-fi para el acceso a la información en la intranet y las redes sociales, las aulas y bibliotecas virtuales u otros recursos más sencillos como power point, diaporamas, repositorios de materiales docentes digitalizados u otros a través de las cuentas habilitadas, así como la utilización de técnicas de estudio que propicien la búsqueda y procesamiento de la información, la presentación y argumentación de los resultados académicos.

- La creación de tradiciones, al menos una tradición, que marque la diferencia y la distinción de cada carrera dentro de la UO, a partir de actividades generales que integre tareas de impacto social intra y extrauniversitarias, acciones de un proyecto comunitario y de investigación vinculado con los problemas de su entorno que lo comprometan con su participación en la solución.

- La construcción de un proyecto de vida que jerarquice la motivación y compromiso con el ejercicio de la profesión y la contribución a la solución de los problemas de su país, su localidad y la universidad.

- La ejercitación de una expresión oral y escrita donde sea capaz de exponer, argumentar, demostrar posiciones, ideas, resultados o soluciones a problemas con elevado dominio de la lengua materna, unido a una adecuada presencia personal en cada contexto.

- El desarrollo de habilidades prácticas en laboratorios y talleres.

- El acceso a las prácticas con el equipamiento y tecnologías que solo existen en las entidades en las que realizan las prácticas.

- El establecimiento de adecuadas relaciones de convivencia en las relaciones interpersonales y la asunción de un comportamiento ético en las actividades que se desarrollan en los espacios no áulicos.

- La creación de actividades para la ampliación de la cultura general que complementen las necesidades docentes, físicas, deportivas, culturales y recreativas como parte de la formación integral.

- La atención diferenciada al 1. año en todas las carreras, años académicos y grupos estudiantiles.

- $\quad$ El desempeño en la realización y sistematización de ejercicios integradores a partir del diseño de la disciplina principal integradora, desde la concepción sistémica de los modos de actuación del profesional en el tránsito por cada año académico y la culminación de estudios, que se manifiestan en la capacitación para la solución de los problemas profesionales en la práctica laboral e investigativa.

- El desarrollo de la formación investigativa para la solución de problemas científicos del objeto de la profesión y su reconocimiento desde el nivel de carrera, por sus resultados y premios producto de la presentación en eventos (concursos, fórums, talleres científicos, congresos, etc.) internacionales, nacionales y territoriales.

De manera general se hace necesario registrar, sistematizar y valorar los resultados e impactos que se van obteniendo sistemáticamente en la labor educativa a todos los niveles, 
a partir de métodos e instrumentos que incluyan los registros de sistematización, la observación participante, encuestas de satisfacción, intercambio, diálogo con los estudiantes y el grupo en los diferentes escenarios educativos con la guía de indicadores para el análisis del cumplimiento como:

$>$ Creatividad, contextualización y personalización de las acciones y actividades previstas y desarrolladas a partir de las estrategias educativas.

$>$ Calidad de los indicadores de promoción alcanzados, rendimiento académico, eficiencia terminal, etc.

> Autogestión del aprendizaje con el uso de las nuevas tecnologías.

$>$ Modos de comportamientos adecuados que manifiestan los estudiantes y docentes.

$>$ Participación y protagonismo estudiantil en las diferentes actividades.

$>$ Niveles de satisfacción de los estudiantes con la labor educativa de sus profesores.

Actualmente, la Universidad de Oriente puede mostrar logros en la labor educativa evidenciados en la progresiva incrementación de carreras que han alcanzado la condición de excelencia y acreditadas por la Junta Nacional de Acreditación. Entre las fortalezas se destacan:

- En la universidad todas las carreras poseen su estrategia educativa, derivada de la institucional, concebida con un enfoque sistémico, que tiene como elemento jerárquico la formación integral de los estudiantes, con énfasis en la labor educativa y con un balance adecuado y concreto de los procesos de pregrado, postgrado, investigación y extensión universitaria.

- Satisfacción de los estudiantes con la calidad de la formación del profesional a partir del reconocimiento al desempeño del claustro en su labor docente metodológica y educativa, expresada en un $95,5 \%$ de satisfacción en las encuestas aplicadas.

- Incremento tendencial de los principales indicadores del proceso de formación de pregrado como la promoción, la eficiencia en el ciclo y la eficiencia académica limpia.

- Se desarrollan estrategias y proyectos que favorecen la convivencia y el protagonismo de la comunidad estudiantil en los diferentes espacios de formación académicos, laborales, recreativos y culturales.

- El movimiento de artistas aficionados y el deporte universitario alcanzan importantes premios en los principales eventos nacionales y se eleva la calidad de los festivales y competencias que se desarrollan con la integración del claustro, las instituciones culturales y deportivas del territorio.

- Es creciente el nivel de desempeño de los estudiantes como consecuencia de la sistematización de los ejercicios integradores que se manifiestan en la capacitación para la solución de los problemas profesionales en la práctica laboral e investigativa.

- El uso de la tecnología disponible favorece la gestión del aprendizaje de los estudiantes, con el mejoramiento de la infraestructura tecnológica desde la red cableada y wi - fi lo que incrementa las potencialidades para el acceso a la información en la intranet y las redes sociales. 
- La creación de tradiciones en las carreras a partir de las tareas de impacto social, relacionadas con el objeto de la profesión en el contexto de las unidades docentes en que se desarrollan las actividades laborales e investigativas y de los proyectos comunitarios, lo que favorecen la formación integral de los estudiantes.

\section{CONCLUSIÓN}

Se necesita continuar la búsqueda, profundización y sistematización de contenidos teóricos, prácticos y metodológicos relacionados con la labor educativa con los jóvenes en la actualidad, con vistas de encontrar las mejores prácticas, maneras, vías y métodos educativos que permitan movilizarlos y comprometerlos intrínsecamente con su formación integral orientados por un claustro preparado académica, pedagógicamente y sensibilizado con esta labor, que se convierte en el corazón de la formación del profesional en Cuba.

Se avanza en el análisis de la formación integral del profesional en la UO desde la experiencia de los claustros de cada carrera, lo que precisa de mayor intencionalidad en el diseño y ejecución de la Estrategia Educativa hasta el año académico y grupo, basado en nuevas perspectivas, proyecciones, pautas y recomendaciones pedagógicas.

Se impone involucrar a toda la comunidad universitaria en la labor educativa, sistemática e intencionada en todos los escenarios de la formación en que se desempeñan los estudiantes.

\section{BIBLIOGRAFÍA}

1. Báxter, E. Educar en valores. (2007). Tarea y reto de la sociedad. Ciudad de La Habana: Pueblo y Educación. Cuba.

2. -------- (2002). La Labor educativa en la escuela: Editorial pueblo y Educación. Cuba.

3. ------ (2003). Cuándo y cómo educar en valores. La Habana: Editorial Pueblo y Educación.

4. Bernstein, B. (1990). La estructura del discurso pedagógico. España: Gedisa

5. Chacón N.y Báxter, E. (2002). Dimensión ética de la Educación Cubana. Ciudad de la Habana: Pueblo y Educación.

6. Egea, M. (2007). Labor Educativa. Selección de lecturas. Ciudad de La Habana: Pueblo y Educación.

7. Colectivo de autores ICCP. (2012). Pedagogía. 3ra. La Habana. Cuba: Editorial Pueblo y Educación.

8. Cubela, JM. (2016). La labor educativa de los jóvenes: un reto de las universidades del siglo XXI. Guayaquil. Ecuador: En Revista Investigación, Formación y Desarrollo. Edición No 3. Enero de. 2016. ISSN 1390-9681.

9. --------- (2016). Proyección estrategia de la labor educativa en la UO Conferencia Científico Metodológica. Santiago de Cuba. Cuba. Con ISBN 978-95913-1137-0. El 22 de abril de 2016.

10. Ferrer Vicente, M (2017). Balance de la formación integral del profesional de pregrado de la Universidad de Oriente. Curso académico 2016 - 2017. Primer semestre. Stgo de Cuba. 
11. Ferrer V, M, Cubela G, JM y Rebollar V. M (2017). El proyecto personal de vida, resultado e impacto de un proyecto educativo inclusivo. Guayaquil. Ecuador: En el 3er Congreso Internacional de Ciencias Pedagógicas. ISBN: 978-9942-17-027-9.

12. Giroux, H. (1997). Los profesores como intelectuales. España: Paidós.

13. Horruitiner S, P. (2000). La labor educativa desde la dimensión curricular. La Habana. Cuba: Pedagogía Universitaria 2000 Vol. 5 No. 1.

14. --- Horruitiner, P. (2009). La universidad cubana: el modelo de formación. La Habana, Cuba: Editorial Universitaria Félix Varela.

15. Makarenko, A. S. Conferencias sobre Educación Infantil. Dirección Provincial de Planes Especiales. Ciudad de la Habana: MINED: Cuba. 1971.

16. Ministerio de Educación Superior. (2015). Junta de Acreditación Nacional. Sistema de Evaluación y Acreditación de Carreras Universitarias (SEA-CU). La Habana, Cuba: Ministerio de Educación Superior.

17. Morin, E. (2000). Los siete saberes necesarios para la educación del futuro. Caracas, CIPOST-UCV-IECSAI-UNESCO.

18. ------------ Los siete saberes necesarios para la educación del futuro. Recuperado: http://unesdoc.unesco.org/images/0011/001177/117740so.pdf- -Vilera G, A. La labor educativa en la sociedad del siglo XXI: Revista de Teoría y Didáctica de las Ciencias Sociales. Mérida-Venezuela. ISSN 1316-9505. Enero-diciembre. № 10 (2005): 133-158.

19. Partido Comunista de Cuba. (2011). Lineamientos de la política económica y social del Partido y la Revolución. La Habana, Cuba: Comité Central del PCC.

20. Partido Comunista de Cuba. (2012). Objetivos de trabajo del Partido Comunista de Cuba aprobados por la Primera Conferencia Nacional. La Habana, Cuba: PCC.

21. Vilera G, A. (2005). La labor educativa en la sociedad del siglo XXI. Revista de Teoría y Didáctica de las Ciencias Sociales. Mérida-Venezuela. ISSN 1316-9505. Enerodiciembre. № 10 (2005): 133-158. 